\title{
Recurrent Orbital Cellulitis Secondary to the 'Forgotten' Scleral Buckle: A Case Report
}

\author{
Hor SM, Noor Aniah A, Mushawiahti M, Malisa A, Bastion MLC ( $\bowtie)$ \\ Department of Ophthalmology, Faculty of Medicine, Universiti Kebangsaan Malaysia Medical Centre, \\ Jalan Yaacob Latif, Bandar Tun Razak, 56000 Cheras, Kuala Lumpur, Malaysia.
}

\begin{abstract}
Scleral buckle placement is a well-established technique for the treatment of primary rhegmatogenous retinal detachment. Complications associated with scleral buckle are uncommon and its presentations can be vary. We report a case of recurrent orbital cellulitis with anterior segment ischemia following a forgotten episode of previous scleral buckling surgery, presenting with blurring of vision, redness and swelling of the lids. The presence of scleral buckle was detected by detailed examination and confirmed by orbital imaging. Orbital infection and rubeosis iridis were successfully treated with scleral buckle removal, intravenous antibiotics and intracameral ranibizumab. However, the retinal detachment recurred and the visual acuity deteriorated to light perception. There was no further intervention as the family declined in view of her old age. In cases of recurrent orbital infection, detailed clinical examination is important to look for evidence of ocular prostheses as a source of infection. Orbital imaging is an adjunct for making the diagnosis especially in cases where history is unreliable. Anterior segment ischemia due to scleral buckle responds well to buckle removal with ranibizumab injection.
\end{abstract}

Keywords: Iris, orbital cellulitis, recurrence, retinal detachment, scleral buckling

\section{Correspondence:}

Mae-Lynn Catherine Bastion. Department of Ophthalmology, Faculty of Medicine, Universiti Kebangsaan Malaysia Medical Centre, Jalan Yaacob Latif, Bandar Tun Razak, 56000 Cheras, Kuala Lumpur, Malaysia. Tel: +603-91457207 Fax: +60391456733 E-mail: maelynnb2003@yahoo.com

\section{Introduction}

Scleral buckling with exoplant has been an effective method in treating primary rhegmatogenous retinal detachment for more than 50 years (1). Long-term complications include induced myopia, diplopia, strabismus, foreign body sensation, infection, extrusion, intrusion, anterior segment, retinal and choroidal ischaemia $(1,2)$. Complications may present years after surgery with different clinical manifestation (1). Infection of scleral buckle remains a significant complication and can results in orbital infection. Almost all cases are resistant to antimicrobial treatment and removal of buckling elements is required for resolution (3). Anterior segment necrosis is another serious complication of scleral buckle. Wilson et al reported up to $8.2 \%$ of eyes had anterior segment necrosis while performing post mortem in eyes with scleral buckle (4).

In this case report, we describe a case of recurrent orbital cellulitis with anterior segment ischemia induced by a forgotten and missed silicone scleral tire from previous retinal detachment surgery.

\section{Case Report}

An 85-year-old woman presented with one-day history of recurrent painless eye redness, yellowish discharge and swelling of the left eye associated with one-month history of blurred vision. She was treated for left orbital cellulitis, 'choroidal detachment' and was investigated for possible underlying malignancy in another eye center one month prior to presentation. 


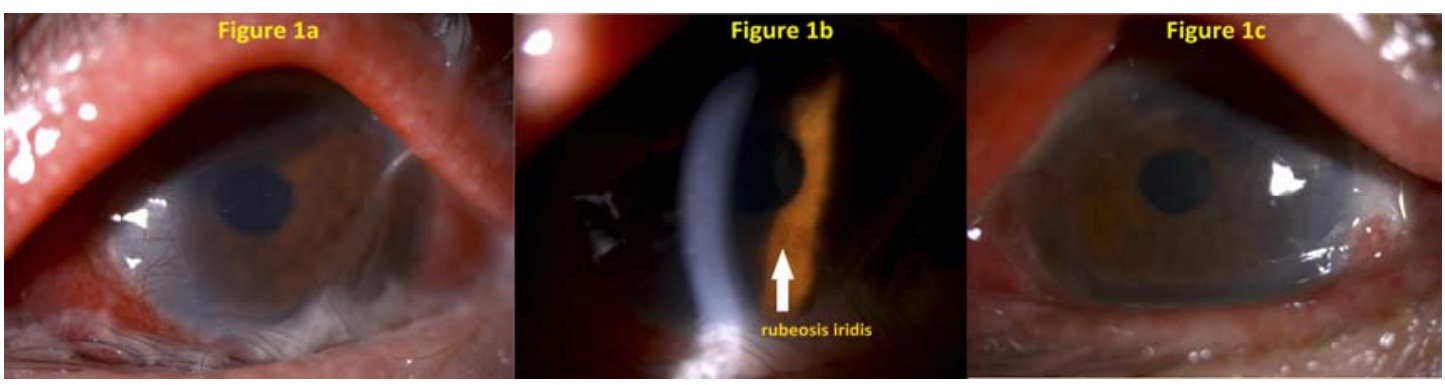

Figure 1: a) Anterior segment photograph of the left eye at presentation. Left conjunctiva was injected, chemosed with corneal vascularization nasally. Mucopurulent discharge with left lower lid entropion were noted. Pupil was rigid and could not be dilated beyond the size shown in the photograph; b) Rubeosis iridis was seen in left eye at the pupil margin in this slit anterior segment photograph during presentation; c) Conjunctiva injection and rubeosis iridis of left eye improved one week after removal of scleral buckle with intracameral ranibizumab.

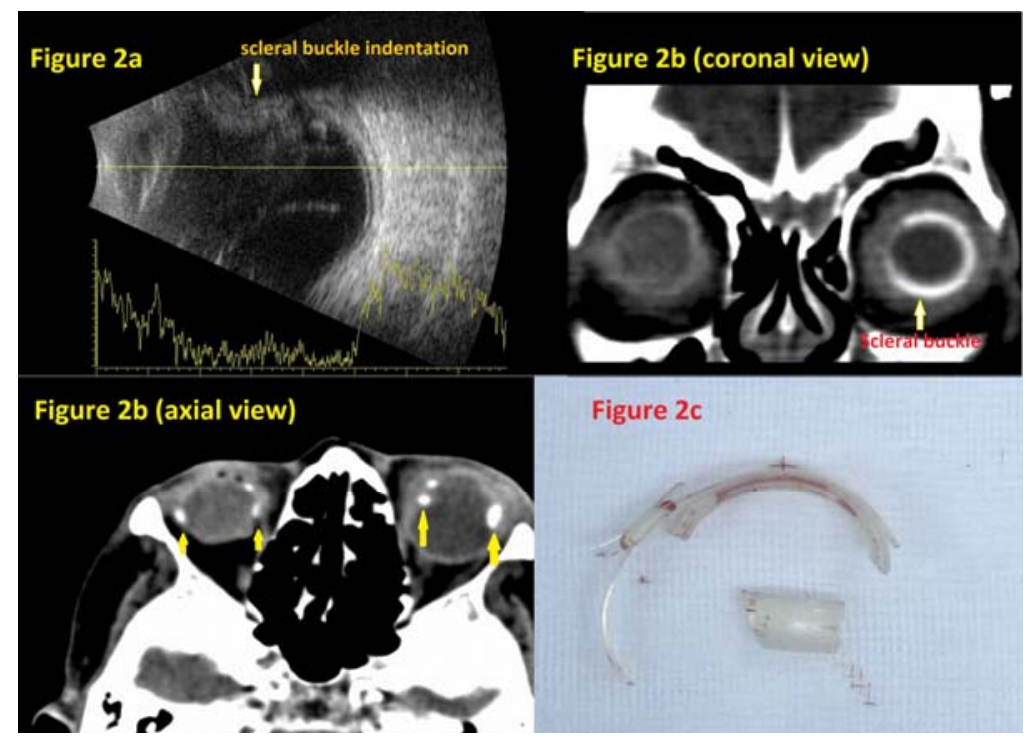

Figure 2: a) Hyperechoic scleral buckle indenting the globe with vitreous opacity; b) Axial and coronal view computed tomography of the orbit showed hyperdense structures (scleral buckle, arrow) encircling both globes with right pthisical bulbi; c) Encircling scleral buckle (silicone tire with Watzke sleeve) which was removed and sent for culture.

She had underlying diabetes mellitus, hypertension, ischemic heart disease, chronic obstructive pulmonary disease and dementia. Cataract extraction was done in both eyes 15 years ago. Her right eye was blind which her family members were unsure of the cause. They were unable to provide any other past ocular or surgical history.

Ocular examination revealed her right vision was no perception to light and left vision was hand movement. Her right eye was pthisical. The left eyelids were swollen with lower lid entropion and mucopurulent discharge (Fig. 1a). The conjunctiva was injected, cornea was hazy, rubeosis iridis was seen with anterior chamber cells of $3+$ (moderate inflammation in between the cornea and iris). Pupil was rigid with maximum dilatation of $3 \mathrm{~mm}$ (Fig. 1b). Left eye was aphakic and the intraocular pressure was $28 \mathrm{mmHg}$.
Gonioscopy was unsuccessful due to the hazy cornea. Fundus examination was further hampered due to the poor pupillary dilatation and cornea haziness. Otherwise systemic examination revealed no breast lumps, hepatomegaly or lymphadenopathy.

Ultrasound brightness (B) scan was performed at presentation which was suggestive of choroidal detachment with nonspecific vitreous opacity (Fig. 2a). Orbital computed tomography (CT) scan was performed and patient was also subjected to extensive investigations to rule out underlying malignancy which predisposed to secondary metastasis to the choroid.

She was treated with intravenous amoxicillin/ clavulanic acid, topical moxifloxacin and topical antiglaucoma (brimonidine and brinzolamide) for the left 
eye. The clarity of the fundus improved three days after treatment as anterior segment inflammation subsided. It was noted that there was presence of regular circumferential retinal indentation in the left eye fundus suggestive of an encircling scleral buckle, rather than a choroidal effusion. Examination of the conjunctiva further detected exposed prolene suture at the temporal conjunctiva. Revision of the orbital CT scans, likewise noted hyperdensities seen in both eyes which were likely to represent prosthesis, most likely encirclage (Fig. 2b).

The scleral buckle was removed and intracameral ranibizumab $0.5 \mathrm{mg} / 0.05 \mathrm{ml}$ was given under general anesthesia. An encirclage comprising of Watzke sleeve and silicone band were identified (Fig. 2c). The buckle bed was flushed with gentamicin. Treatment with intravenous amoxicillin/clavulanic acid and topical moxifloxacin was continued post-operatively.

The rubeosis iridis was completely resolved by day one post-operation while the inflammation and discharge were markedly reduced by day three postremoval of buckle (Fig. 1c). Scleral buckle microscopic examination indicated gram positive cocci. However, there was no growth from the buckle itself. Unfortunately, one-week post-removal of scleral buckle, her retina re-detached and her vision deteriorated to light perception. The family declined further intervention due to her age and risks of general anaesthesia. She was reviewed 9 weeks after single dose of ranibizumab and the rubeosis iridis did not recur. However, the final visual acuity remained light perception.

\section{Discussion}

Scleral buckle placement is an effective method to reattach the retina and to preserve central vision for primary rhegmatogenous retinal detachment (1). Scleral buckle elements are usually left in place and removed when only complication arises. Most commonly reported complications are extrusion of buckle and infection (1). Common signs and symptoms include pain, discomfort, irritation, redness, chemosis and discharge. Diagnosis is evident if patients are able to offer past history of scleral buckling or surgical notes are available.

In our case, the diagnosis of infected scleral buckle was not made during first presentation resulting in recurrent orbital infection. Thus, thorough examination is important to look for the possible underlying problem especially in a case of recurrent orbital cellulitis such as exposed sutures in this case. In cases with poor fundus view due to rigid pupil or hazy cornea, buckle indentation can be misleading as choroidal detachment. Ultrasound B scan on presentation was interpreted as 'choroidal detachment' with vitreous opacity. Solid silicone buckle appears hyperechoic and indenting the globe in ultrasound B scan (5). On the other hand, serous detachment of choroid appears as an echolucent region while haemorrhagic detachment is seen as dense hyperechoic region within the suprachoroidal space (6). The shape of the globe is preserved in choroidal detachment unlike scleral buckling. Solid silicone buckling elements are uniformly hyperdense at CT and appears as an encircling band that surrounds the globe (7). Imaging is useful in evaluating complications post scleral buckling in cases whereby patients are unable to provide relevant history and where surgical notes are unavailable.

Various organisms had been cultured from removed scleral buckling elements and the most frequently isolated bacteria were $S$. epidermidis, coagulasenegative staphylococci, S. aureus, corynebacteria, Mycobacterium chelonei, and Proteus mirabilis (8). Staphylococcus aureus is a common colonizer of the eyelid margins and frequently causes blepharitis or orbital cellulitis (9). Microscopy examination in our patient showed gram positive cocci which was likely to be skin flora, however culture yielded no growth. This is probably due to prior antimicrobial treatment. Holland et al. demonstrated bacterial biofilms on surfaces and end of both silicone sponge and solid silicone buckling elements which were removed following infection and extrusion (3). The biofilms enhance adherence and colonization of bacteria onto prosthetic devices like scleral buckle, it also protects against host defenses mechanism and limit the penetration of antimicrobial therapy (3). Thus, removal of an infected scleral buckle is necessary for resolution of infection. We believe that recurrence of orbital cellulitis in our patient was attributed to the above biofilm mechanism. Although medical treatment is the first-line treatment for infected scleral buckle, our patient underwent scleral buckle removal after intravenous antimicrobial to eliminate the source of infection as the infection was intractable.

Anterior segment ischemia is another rare complication of scleral buckling. It can lead to rubeosis iridis and subsequently neovascular glaucoma. Factors causing anterior segment ischemia after scleral buckling include damage to vortex vein, disruption of anterior ciliary arteries and long posterior ciliary arteries $(1,10)$. Systemic conditions such as age, atherosclerosis, and hematological disorders including anemia, hypercoagulability or sickle cell disease are contributing factors (1). Long ciliary arteries could be 
disrupted by cryotherapy or compression from scleral buckle (10). Occlusion of vortex vein through buckle compression can cause ischaemia (11).

We believe that anterior segment ischemia in our patient is due to atherosclerosis and buckle compression of uveal blood flow. The rubeosis iridis responded well to scleral buckle removal and a single intracameral ranibizumab injection. She was reviewed 9 weeks after single dose of ranibizumab and the rubeosis iridis remained regressed suggesting a direct link between a tight buckle and her initial rubeosis. Other signs of anterior segment ischemia which was seen in this patient were anterior chamber reaction, ischemic iris dilator pupillae, mild cornea edema, rubeosis iridis and raised intraocular pressure. Signs not seen in this patient are hyphema, striate keratopathy, iris atrophy and ocular hypotony (12). Anterior segment ischemia is more common in encircling buckles than segmental buckles. Hence segmental buckles should be used whenever possible. The treatment of anterior segment ischemia is not necessarily removal of the buckle. Instead, the encircling element can be cut to allow some of the indentation to be retained. Unfortunately, this was not an option for our patient who had signs of buckle infection.

\section{Conclusion}

Scleral buckle infection can manifest as orbital cellulitis. Thorough clinical examination in conjunction with orbital imaging are important to look for evidence of ocular prostheses in cases of recurrent orbital infection when history is unreliable. Anterior segment ischemia due to scleral buckle responds well to scleral buckle removal with ranibizumab injection.

\section{References}

1. Tsui I. Scleral buckle removal: indications and outcomes. Surv Ophthalmol 2012;57(3):253-63.

2. Schwartz SG, Kuhl DP, McPherson AR, Holz ER, Mieler WF. Twenty-year follow-up for scleral buckling. Arch Ophthalmol. 2002;120(3):325-9.

3. Holland SP, Pulido JS, Miller D, et al. Biofilm and scleral buckle-associated infections: a mechanism for persistence. Ophthalmology. 1991;98(6):933-8.
4. Wilson DJ, Green WR. Histopathologic study of the effect of retinal detachment surgery on 49 eyes obtained post mortem. Am J Ophthalmol. 1987;103(2):167-79.

5. Kanoff J, Ginat DT, Singh A, Kim I. In: Ginat DT, Freitag SK (eds) Post-treatment imaging of the orbit. Imaging after vitreoretinal surgery. Berlin, Heidelberg: Springer, 2015, pp-153-69.

6. Yeh PT. B-scan for the diagnosis of choroidal disease. Journal of Medical Ultrasound. 2013;21(3):123-125.

7. Lane JI, Watson RE, Witte RJ, McCannel CA. Retinal detachment: imaging of surgical treatments and complications. Radiographics. 2003;23(4):983-94.

8. Holland SP, Pulido JS, Miller D, et al. Biofilm and scleral buckle-associated infections, a mechanism for persistence. Ophthalmology. 1991;98(6):933-8.

9. Nakata K, Inoue Y, Harada J, et al. A high incidence of Staphylococcus aureus colonization in the external eyes of patients with atopic dermatitis. Ophthalmology. 2000;107(12):216771.

10. Freeman HM, Hawkins WR, Schepens CL. Anterior segment necrosis. An experimental study. Arch Ophthalmol. 1966;75(5):644-50.

11. Janssens K, Zeyen T, Van Calster J. Anterior segment ischemia with rubeosis iridis after a circular buckling operation treated successfully with an intravitreal bevacizumab injection: a case report and review of the literature. Bull Soc Belge Ophtalmol. 2012;(319):5-9.

12. Saunders RA, Bluestein EC, Wilson ME, Berland JE. Anterior segment ischemia after strabismus surgery. Surv Ophthalmol. 1994;38(5):456-66. 